П. М. Тамразов, С. А. Охрименко (Ин-т математики НАН Украниы, Киев)

\title{
ПАРНЫЕ ПРОИЗВЕДЕНИЯ МОДУ ЛЕЙ СЕМЕЙСТВ КРИВЫХ НА РИМАНОВОМ ЛИСТЕ МЕБИУСА"
}

Pairwise products of moduli of families of curves on a Riemannian Möbius strip are investigated and estimates for these products are obtained. As one of factors, the modulus of a family of arcs is considered belonging to a wide class of families of this sort, for which the moduli and the extremal metrics are also found.

Досліджено парні добутки модулів сімей крнвнх на рімановому лнстку Мьобіуса та одержані оцінки для цих добутків. Як один із мпожників розглядається модуль сім'ї дуг з широкого класу таких сімей (і для кожної з них паһ̆дено модуль та екстремалыну метрику).

1. Введение. Оценки парных произведений модулей сопряженных семейств кривых играют важную (часто решающую) роль в многочисленных приложенияХ. В данной работе вопрос о таких оценках рассматривается для неориентируемого многообразия. В качестве одного из компонентов пары выстутает произвольное гомотопическое семейство нестягиваемых петель на римановом листе Мебиуса или произвольное объединение таких семейств. Модули этих семейств найдены ранее [1 - 3]. В качестве сопряженного компонента пары выступает семейство дуг из широкого класса семейств, и для каждого из них вычислен модуль (для одного из таких семейств модуль был указан ранее в [4]). Полученные нами оценки произведений модулей являются усилением и обобщением основного результата из [4] о нижней оценке одной из характеристик риманова листа Мебиуса (см. ниже).

Пусть $0<\alpha \leq+\infty, 0<\beta<+\infty$. В дальнейшем используем следующие понятия и обозначения из [1-3]: множество

$$
\Pi_{0, \alpha}:=\{(x, y):-\alpha<x<\alpha,-\beta \leq y<\beta\}
$$

в $R^{2}$, риманов лист Мебиуса $\Pi_{\alpha}$, класс $P\left(\Pi_{\alpha}\right)$ метрик $\rho$ в $\Pi_{\alpha}$, функционал $A_{\alpha}(\rho)$, конкретную петлю $\gamma^{1} \subset \Pi_{\alpha}$, семенство $\Gamma_{l, \alpha}$ петель на $\Pi_{\alpha}$, понятия экстремальной метрики и модуля семейства кривых на $\Pi_{\alpha}$.

2. Модули поперечных семейств дуг. Пусть $0<\alpha<+\infty$.

Дугой в $\Pi_{\alpha}$ условимся называть непрерывное отображение непустого открытого действительного интервала в $\Pi_{\alpha}$.

Пусть $a<b$ и $\delta:(a, b) \rightarrow \Pi_{\alpha}-$ дуга в $\Pi_{\alpha}$. Положим $c=\frac{a+b}{2}$ и через $\delta^{-}$ и $\delta^{+}$условимся обозначать сужения отображения $\delta$ соответственно на полуинтервалы $(a, c]$ и $[c, b)$. Отображения $\delta^{+}$и $\delta^{-}$условимся называть полудугальи, причем те же обозначения и термин будем применять и к соответствующим образам указанных полуинтервалов. Ниже на дуги $\delta$ накладываем некоторые комбинащии следующих условий.

I. Для всякого компакта $K \subset \Pi_{\alpha}$ сушествует компакт $T \subset(a, b)$ такой, что $\delta(t) \notin K \forall t \in(a, b) \backslash T$.

II. Существует компакт $K_{1} \subset \Pi_{\alpha}$ такой, что: каков бы ни был компакт, $T_{1} \subset(a, b)$, на $(a, b) \backslash T_{1}$ существуют точки $a_{1}$ и $b_{1}>a_{1}$, для которых всякая дуга в $\Pi_{\alpha}$ с началом $\delta\left(a_{1}\right)$ и концом $\delta\left(b_{1}\right)$, гомотопная в $\Pi_{\alpha}$ дуге $\delta:\left(a_{1}\right.$, $\left.b_{1}\right) \rightarrow \Pi_{\alpha}$, имеет непустое пересечение с $K_{1}$.

\footnotetext{
* Выполнена при частичноһ поддержке Государственного фонда фундамеитальных исследования при Мипистерстве Укранны по делам пауки и технологић (проект 1.4/263) и INTAS (грaнt 94-1474).
} 
III. Множества $\delta((a, c])$ и $\delta([c, b))$ не являются относительно компактными в $\Pi_{\alpha}$.

IV. Дуга $\delta$ имеет непустое пересечение с петлей $\gamma^{1}$.

Обозначим через $B^{\alpha}$ семейство всех дуг в $\Pi_{\alpha}$, удовлетворяющих условиям III и IV, а через $B_{\alpha}$ - семейство всех жордановых дуг в $\Pi_{\alpha}$ вида $\delta_{y}$ : $(-\alpha, \alpha) \rightarrow \Pi_{\alpha}$, задаваемых условием $\delta_{y}(t)=(t, y) \in \Pi_{\alpha}$ или $\delta_{y}(t)=(-t, y) \epsilon$ $\in \Pi_{\alpha}$, где $y$ - параметр, принимающий любое постоянное значение из промежутка $[-\beta, \beta)$.

Обозначим через $B_{0}^{\alpha}$ семейство всех дуг в $\Pi_{\alpha}$, удовлетворяющих условиям I и II.

Можно убедиться, что $B_{\alpha} \subset B_{0}^{\alpha} \subset B^{\alpha}$.

Заметим, что рассмотренное в [4] семейтво $B_{1}^{\alpha}$ дуг, имеющих индекс пересечения \pm 1 с каждой кривой из семеиства $\Gamma_{1, \alpha}$, удовлетворяет условию $B_{\alpha} \subset$ $\subset B_{1}^{\alpha} \subset B_{0}^{\alpha}$ (в [4] для $B_{1}^{\alpha}$ использовано иное обозначение).

Пусть теперь $\Gamma_{\alpha}^{*}$ - произвольное семейство локально спрямляемых дуг в $\Pi_{\alpha}$, удовлетворяющее условиям $B_{\alpha} \subset \Gamma_{\alpha}^{*} \subset B^{\alpha}$.

Используя комплексную переменную $w=x+i y$, установим следущий результат.

Теорема 1. $B \Pi_{\alpha}$ метрика

$$
\rho_{\alpha}^{*}(w):=\frac{1}{2 \alpha} \forall w \in \Pi_{\alpha}
$$

экстрельальна для селейства $\Gamma_{\alpha}^{*}$ и $M\left(\Gamma_{\alpha}^{*}\right)=\frac{\beta}{\alpha}$.

Доказательство. Сначала докажем допустимость метрики $\rho_{\alpha}^{*}$ для семейства $B^{\alpha}$. Через $\Pi_{\alpha}^{2}$ обозначим двулистное накрывающее многообразия $\Pi_{\alpha}$, реализованное в комплексной плоскости $\mathrm{C}$ в виде кольца:

$$
\Pi_{\alpha}^{2}:=\left\{z \in \mathrm{C}, e^{-\varphi}<|z|<e^{\varphi}\right\}, \quad \varphi:=\frac{\pi \alpha}{2 \beta},
$$

с проектированием $p: \Pi_{\alpha}^{2} \rightarrow \Pi_{\alpha}, p(1)=0$ и группой скольжений $Z_{2}$, образующая которой является антиконформным гомеоморфизмом $z \mapsto-\frac{1}{\bar{z}}$.

Пусть $\delta:(a, b) \rightarrow \Pi_{\alpha}$ - дуга из $B^{\alpha}$. Без ограничения общности считаем, что $a=-1, b=1$. Пусть $\delta^{+}:=\left.\delta\right|_{[0,1)}$, а $\delta_{1}^{+}, \delta_{2}^{+}$一 поднятия на $\Pi_{\alpha}^{2}$ полудуги $\delta^{+}$. Тогда $\delta_{1}^{+}, \delta_{2}^{+}$не являются относительно компактными в $\Pi_{\alpha}^{2}$ и переходят друг в друга при отображении $z \mapsto-\frac{1}{\bar{z}}$. Аналогичные утверждения справедливы и по отношению к полудуге $\delta^{-}:=\left.\delta\right|_{(-1,0]}$ и ее поднятиям $\delta_{1}^{-}, \delta_{2}^{-}$на $\Pi_{\alpha}^{2}$. При этом дуги $\delta_{1}^{-} \cup \delta_{1}^{+}$и $\delta_{2}^{-} \cup \delta_{2}^{+}$пересекают окружность $|z|=1$, так как она является полным прообразом петли $\gamma^{1}$ в отображении $p: z \mapsto w$. Имеем

$$
\left|p^{\prime}(z)\right|=\left|\left(\frac{2 \beta}{\pi} \log z\right)^{\prime}\right|=\frac{2 \beta}{\pi|z|}, \quad|d w|=\frac{2 \beta}{\pi}|d \log z|,
$$

поэтому 


$$
\int_{\delta}|d w|=\frac{2 \beta}{\pi} \int_{\delta_{1}^{-} \cup \delta_{1}^{+}}|d \log z| \geq \frac{2 \beta}{\pi} \int_{\delta_{1}^{-} \cup \delta_{1}^{+}}|d \operatorname{Re} \log z| \geq 2 \alpha .
$$

Следовательно, в $\Pi_{\alpha}$ метрика $\rho_{\alpha}^{*}(w)=(2 \alpha)^{-1}$ допустима для семейства $B^{\alpha}$, а поэтому и для $\Gamma_{\alpha}^{*} \subset B^{\alpha}$. При этом

$$
A_{\alpha}\left(\rho_{\alpha}^{*}\right)=\frac{1}{4 \alpha^{2}} 4 \alpha \beta=\frac{\beta}{\alpha} .
$$

Значит,

$$
M\left(B^{\alpha}\right) \leq \frac{\beta}{\alpha} .
$$

Пусть теперь $\rho$ - произвольная допустимая для $B_{\alpha}$ метрика. Тогда последовательно имеем

$$
\begin{gathered}
\iint_{\Pi_{\alpha}} \rho d x d y=\int_{-\beta}^{\beta}\left(\int_{-\alpha}^{\alpha} \rho d x\right) d y \geq \int_{-\beta}^{\beta} 1 d y=2 \beta \\
\left(\iint_{\Pi_{\alpha}} \rho d x d y\right)^{2} \leq A_{\alpha}(\rho) A_{\alpha}(1)=4 \alpha \beta A_{\alpha}(\rho) ; \\
(2 \beta)^{2} \leq\left(\iint_{\Pi_{\alpha}} \rho d x d y\right)^{2} \leq 4 \alpha \beta A_{\alpha}(\rho) \\
A_{\alpha}(\rho) \geq \frac{\beta}{\alpha} .
\end{gathered}
$$

Значит,

$$
M\left(B_{\alpha}\right) \geq \frac{\beta}{\alpha}
$$

Поскольку $B_{\alpha} \subset \Gamma_{\alpha}^{*} \subset B^{\alpha}$, то

$$
M\left(B_{\alpha}\right) \leq M\left(\Gamma_{\alpha}^{*}\right) \leq M\left(B^{\alpha}\right) .
$$

Сопоставляя (1) - (3), получаем

$$
M\left(B_{\alpha}\right)=M\left(\Gamma_{\alpha}^{*}\right)=M\left(B^{\alpha}\right)=\frac{\beta}{\alpha} .
$$

Следовательно, метрика $\rho_{\alpha}^{*}(w)=(2 \alpha)^{-1}$ экстремальна для каждого из семейств $B_{\alpha}, \Gamma_{\alpha}^{*}, B^{\alpha}$. Теорема 1 доказана.

3. Произведения модулей и их оценки. В настоящем пункте $\Gamma_{0, \alpha}, \Gamma_{1, \alpha}^{k}$, $T_{s, m}$ обозначают введенные в [1 - 3]семейства петель $(k, s, m$-натуральные).

В [1 - 3] найдены экстремальные метрики и модули семейств $\Gamma_{0, \alpha}, \Gamma_{1, \alpha}^{k}$, $T_{s, m}$. Ниже сушественно используем эти результтаты.

Введем обозначение

$$
\varphi:=\frac{\pi \alpha}{2 \beta}
$$

Нами установлены следуюшие результаты о парных произведениях модулей, в которых th обозначает гиперболический тангенс. 
Tеорема 2. В обозначениях

$$
\begin{aligned}
\mathcal{N}_{0} & :=\mathcal{N}_{0}(\alpha):=M\left(\Gamma_{0, \alpha}\right) M\left(\Gamma_{\alpha}^{*}\right), \\
c & :=\log (2+\sqrt{3})(\approx 1,317)
\end{aligned}
$$

справедливы соотношения

$$
\mathcal{N}_{0}= \begin{cases}\frac{1}{\varphi} \text { th } \varphi \geq \frac{1}{c} \text { th } c=\frac{\sqrt{3}}{2 \log (2+\sqrt{3})}(\approx 0,658) & \forall \varphi \leq c, \\ \frac{1}{4 \varphi}(4 \text { th } c-c)+\frac{1}{4}\left(>\frac{1}{4}\right) & \forall \varphi>c\end{cases}
$$

и $\mathcal{N}_{0}$ - строго убываючая функция от $\varphi$ со значенияльи в интервале $(1 / 4,1)$, причемь

$$
\begin{aligned}
& \mathcal{N}_{0} \searrow \frac{1}{4} \text { npu } \varphi \nearrow+\infty, \\
& \mathcal{N}_{0} \nearrow 1 \text { nрu } \varphi \searrow 0 .
\end{aligned}
$$

Tеорема 3. В обозначениях

$$
\mathcal{N}_{k}:=\mathcal{N}_{k}(\alpha):=M\left(\Gamma_{1, \alpha}^{k}\right) M\left(\Gamma_{\alpha}^{*}\right), \quad k=12, \ldots,
$$

справедливы соотношения:

$$
\begin{gathered}
\mathcal{N}_{2 m}=\frac{1}{4 m^{2}} \quad \forall m=1,2, \ldots ; \\
\mathcal{K}_{2 s-1}=\frac{1}{(2 s-1) \varphi} \text { th } \frac{\varphi}{2 s-1}<\frac{1}{(2 s-1)^{2}} \quad \forall s=1,2, \ldots,
\end{gathered}
$$

$u(2 s-1)^{2} \mathcal{N}_{2 s-1}-$ строго убывающая функция от $\frac{\varphi}{2 s-1}$ со зноченияхи в интервале $(0,1)$, причем

$$
\begin{aligned}
& (2 s-1)^{2} \mathcal{N}_{2 s-1} \searrow 0 \text { npu } \frac{\varphi}{2 s-1} \nearrow+\infty, \\
& (2 s-1)^{2} \mathcal{N}_{2 s-1} \nearrow 1 n p u \frac{\varphi}{2 s-1} \searrow 0 .
\end{aligned}
$$

В частности, $\mathcal{N}_{2}=1 / 4$, а $\mathcal{N}_{1}-$ строго убываюшал функция от $\varphi$ со значенияли в интервале $(0,1)$, причем

$$
\begin{gathered}
\mathcal{K}_{1}=\frac{1}{\varphi} \text { th } \varphi, \\
\mathcal{K}_{1} \nearrow_{1} \text { npu } \varphi \searrow 0, \\
\mathcal{N}_{d} \searrow 0 \text { npu } \varphi \nearrow+\infty .
\end{gathered}
$$

Теорема 4. В обозначениях

$$
\begin{gathered}
\mathcal{K}_{s, m}:=\mathcal{K}_{s, m}(\alpha):=M\left(T_{s, m}\right) M\left(\Gamma_{\alpha}^{*}\right), \\
l_{s, m}:=\log \left(\frac{2 m}{2 s-1}+\sqrt{\left(\frac{2 m}{2 s-1}\right)^{2}-1}\right)
\end{gathered}
$$

өерно следуюшее. Если $m<s$, mо $\mathcal{K}_{s, m}=\frac{1}{4 m^{2}}$. Пусть теперь $m \geq s$. Если $\varphi /(2 s-1) \leq l_{s, m}, m o$ 


$$
\mathcal{K}_{, m}=\frac{1}{(2 s-1) \varphi} \text { th } \frac{\varphi}{2 s-1}
$$

u $(2 s-1)^{2} \mathcal{K}_{, m}-$ строго убывающал функция от $\frac{\varphi}{2 s-1}$ со значенияліи в инmepeane $(0,1)$, причелt

$$
(2 s-1)^{2} \mathcal{K}_{, m} \searrow 0 \text { npu } \frac{\varphi}{2 s-1} \nearrow+\infty
$$

$u$

$$
(2 s-1)^{2} \mathcal{N}_{s, m} \nearrow_{1} \quad \text { npu } \frac{\varphi}{2 s-1} \searrow 0
$$

Eсли же $\frac{\varphi}{2 s-1} \geq l_{s, m}$, mo:

1) $0<2 m\left(\mathcal{N}_{s, m}-\frac{1}{4 m^{2}}\right) \varphi=\frac{2 m}{2 s-1}$ th $l_{s, m}-\frac{2 s-1}{2 m} l_{s, m}$ и $2 m\left(\mathcal{N}_{s, m}-\frac{1}{4 m^{2}}\right) \varphi-$ строго растущал функция от $\frac{2 m}{2 s-1}$ со значенияльи в интервале $(0,+\infty)$, причем

$$
2 m\left(\mathcal{K}_{s, m}-\frac{1}{4 m^{2}}\right) \varphi \searrow 0 n p u \frac{2 m}{2 s-1} \searrow 1 ;
$$

2) $\quad$ i $<4 m^{2} \mathcal{N}_{s, m} \leq\left(\frac{2 m}{2 s-1}\right)^{2} \frac{\text { th } l_{s, m}}{l_{s, m}}$

$u$

$$
4 m^{2} \mathcal{N}_{s, m} \rightarrow 1 \quad n p u \quad \frac{2 m}{2 s-1} \searrow 1
$$

3) $0<\left(\mathcal{N}_{s, m}-\frac{1}{4 m^{2}}\right)(2 s-1) \varphi=$ th $l_{s, m}-\left(\frac{2 s-1}{2 m}\right)^{2} l_{s, m}$ $u\left(\mathcal{N}_{s, m}-\frac{1}{4 m^{2}}\right)(2 s-1) \varphi-$ строго растущал функция от $\frac{2 m}{2 s-1}$ со значениями в интервале $(0,1)$, причель

$$
\left(\mathcal{N}_{s, m}-\frac{1}{4 m^{2}}\right)(2 s-1) \varphi \nearrow 1 \text { npu } \frac{2 m}{2 s-1} \nearrow+\infty .
$$

Замечание. В теоремах $2-4$ зависимость величнн $\mathcal{N}_{0}, \mathcal{N}_{k}, \mathcal{N}_{s, m}$ от конформной (и метрической) структуры $\Pi_{\alpha}$ сосредоточена только в параметре $\varphi$ (все остальные параметры зависят лишь от числовых индексов, выбранных на $\Pi_{\alpha}$ семейств кривых).

Для метрики $\rho \in P\left(\Pi_{\alpha}\right)$ введем следуюшие обозначения:

$$
\begin{gathered}
L_{0}(\alpha, \rho):=\inf \left\{\int_{\gamma_{0}} \rho d s: \gamma_{0} \in \Gamma_{0, \alpha}\right\}, \\
L_{k}(\alpha, \rho):=\inf \left\{\int_{\gamma_{k}} \rho d s: \gamma_{k} \in \Gamma_{1, \alpha}^{k}\right\}, \quad k=1,2, \ldots, \\
L^{*}(\alpha, \rho):=\inf \left\{\int_{\gamma^{*}} \rho d s: \gamma^{*} \in \Gamma_{\alpha}^{*}\right\}
\end{gathered}
$$

и для $n=0,1,2, \ldots$ положим 


$$
\begin{gathered}
\Lambda_{n}^{*}(\alpha):=\sup \left\{\frac{L_{n}(\alpha, \rho) L^{*}(\alpha, \rho)}{A_{\alpha}(\rho)}: \rho \in P\left(\Pi_{\alpha}\right)\right\} \\
\mathcal{M}_{n}^{*}(\alpha):=\frac{1}{\Lambda_{n}^{*}(\alpha)} .
\end{gathered}
$$

Легко проверить, что

$$
\left(\mathcal{M}_{n}^{*}(\alpha)\right)^{2} \geq \mathcal{N}_{n}(\alpha) \forall n=0,1,2, \ldots,
$$

а с помощью результатов из [2, 3] и теорем $1-3$ могут быть установлены следуюцие утверждения.

Teopeмa 5. $\left(\mathcal{M}_{n}^{*}(\alpha)\right)^{2}>\mathcal{K}_{n}(\alpha) \forall n=0,1,2, \ldots$

Теорема 6. $\mathcal{M}_{0}^{*}(\alpha)>\sqrt{\mathscr{N}_{0}(\alpha)}>\frac{1}{2} \quad \forall \alpha \in(0,+\infty)$,

$$
\inf _{\alpha} \mathcal{M}_{0}^{*}(\alpha)=\inf _{\alpha} \sqrt{\mathcal{K}_{0}(\alpha)}=\frac{1}{2}
$$

В работе [4] введены и вычислены величины, аналогичные $\mathcal{M}_{0}^{*}(\alpha)$ и $\mathcal{M}_{1}^{*}(\alpha)$ (в менее общих предположениях и иных обозначениях), и показано, что первая из них $>1 / 2$ для всех $\alpha \in(0,+\infty)$, причем постоянная $1 / 2$ неулучшаема. Это основной результат работы Блаттера [4]. Теорема 6 является усилением и обобщением этого результата.

Доказательство теорем $2-4$ опирается на теорему 1 , результаты из $[2,3]$ и следуюшую ниже лемму, в которой обозначено

$$
l(t):=\log \left(t+\sqrt{t^{2}-1}\right) \forall t>1 \text {. }
$$

Ниже ch и sh обозначают гиперболические косинус и синус соответственно.

Лемма. Справедливы соотночения

$$
\begin{aligned}
& \left(\frac{\text { th } t}{t}\right)^{\prime}<0 \quad \forall t>0, \quad 0<\frac{\text { th } t}{t}<1 \quad \forall t>0, \\
& \frac{\text { th } t}{t} \nearrow 1 \text { npu } t \searrow 0 \text {, } \\
& \frac{\text { tht }}{t} \searrow 0 \text { npu } \quad t \nearrow+\infty \\
& \left(t \operatorname{th} l-\frac{l}{t}\right)^{\prime}>0 \quad \forall t>1 \text {, } \\
& t \text { th } l-\frac{l}{t} \searrow 0 \quad n p u \quad t \searrow 1 \text {; } \\
& \left(\frac{t^{2} \mathrm{th} l}{l}\right)^{\prime}>0 \quad \forall t>1 \text {, } \\
& \frac{t^{2} \text { th } l}{l} \searrow 1 \text { npu } t \searrow 1 \text {; } \\
& \left(\text { th } l-\frac{l}{t^{2}}\right)^{\prime}>0 \quad \forall t>1, \quad 0<\operatorname{th} l-\frac{l}{t^{2}}<1 \quad \forall t>1 \text {, } \\
& \text { th } l-\frac{l}{t^{2}} \lambda 1 \text { npu } \quad t \lambda+\infty \text {. }
\end{aligned}
$$


Доказательство. Имеем

$$
\begin{gathered}
\operatorname{ch} l=\operatorname{ch} \log \left(t+\sqrt{t^{2}-1}\right)=\frac{1}{2}\left(\left(t+\sqrt{t^{2}-1}\right)+\left(t+\sqrt{t^{2}-1}\right)^{-1}\right)=t, \\
l^{\prime}=\left(\sqrt{t^{2}-1}\right)^{-1}=(\operatorname{sh} l)^{-1} .
\end{gathered}
$$

При $t>0$ верны соотношения $(\operatorname{sh} t)^{\prime}=\operatorname{ch} t>1=t^{\prime}$, а в точке $t=0$ функции $t$ и $\operatorname{sh} t$ равны. Поэтому $t<\operatorname{sh} t \forall t>0$ и

$$
\left(\frac{\text { th } t}{t}\right)^{\prime}=\frac{t(\operatorname{ch} t)^{-2}-\text { th } t}{t^{2}}=\frac{2 t-\operatorname{sh} 2 t}{2 t^{2} \operatorname{ch}^{2} t}<0 \forall t>0 .
$$

Кроме того, имеем

$$
\lim _{t \rightarrow+\infty} \frac{\text { tht }}{t}=0, \quad \lim _{t \rightarrow 0} \frac{\operatorname{th} t}{t}=\lim _{t \rightarrow 0} \frac{\operatorname{th}^{\prime} t}{t^{\prime}}=\lim _{t \rightarrow 0}(\operatorname{ch} t)^{-2}=1 .
$$

Значит, $0<\frac{\text { th } t}{t}<1 \quad \forall t>0$.

Далее, при $t>1$ имеем

$$
\begin{gathered}
\left(\frac{t^{2} \text { th } l}{l}\right)^{\prime}=\left(\frac{\operatorname{sh} 2 l}{2 l}\right)^{\prime}=\left(\frac{d}{d 2 l}\left(\frac{\operatorname{sh} 2 l}{2 l}\right)\right)\left(2 l^{\prime}\right)>0 \quad \forall t>1, \\
\left(\operatorname{th} l-\frac{l}{t^{2}}\right)^{\prime}=l^{\prime}(\operatorname{ch} l)^{-2}+2 t^{-3} l-t^{-2} l^{\prime}=l^{\prime} t^{-2}+2 t^{-3} l-t^{-2} l^{\prime}= \\
=2 t^{-3} l>0 \quad \forall t>1, \\
\lim _{t \rightarrow \infty}\left(\text { th } 1-\frac{l}{t^{2}}\right)=1,
\end{gathered}
$$

a $\operatorname{tak} \operatorname{kax}\left(t\right.$ th $\left.l-\frac{l}{t}\right)=t\left(t\right.$ th $\left.l-\frac{l}{t^{2}}\right)$, то

$$
\begin{gathered}
\left(t \operatorname{th} l-\frac{l}{t^{2}}\right)^{\prime}>0 \forall t>1, \\
\lim _{t \rightarrow 1}\left(t \text { th } l-\frac{l}{t}\right)=0 .
\end{gathered}
$$

Лемма доказана.

Результаты данной работы первоначально опубликованы в виде препринTa [5].

1. Тахразов П. М. Методы исследования экстремальных метрнк и модулей семейств кривых в „скрученном" римановом многообразии // Мат. сб. - 1992. - 183, № 3. - С. $55-75$.

2. Тамразов П. М. Модули и экстремальные метриқи в скрученных римановых многобразиях // Модули неориентируемых и скрученных рнмановых многообразий. - Кнев, 1997. С. 3-25. - (Препрннт / НАН Укранны. ИН-Т математики; 97.9):

3. Тамразов $П . M$. Модули и экстремальные метрикн в неорнентируемых и скрученных рнмановых многообразнях // Укр. мат. журн. - 1998. - 50, № 10. - С. 1388-1398.

4. Blatter C. Zur Riemannschen Geometrie im Grosse auf dem Mobiusband // Compos. math. 1960. - 15, N 1. - P. 88- 107.

5. Охрименко С. А., Тамразов П.М. Оценки произведеннй модулей семейств кривых на римановом листе Мебиуса // Модули неориентируемых и схрученных рнмановых многообразни. - Кнев, 1997. - С. 26-40. - (Препринт / НАН Украины. Ин-т математикн; 97.9).

Палучено 07.07.97 